\title{
SERIES EXPANSIONS OF THE ANALYTIC FEYNMAN INTEGRAL FOR THE FOURIER-TYPE FUNCTIONAL
}

\author{
Il Yong Lee ${ }^{a}$, Hyun Soo Chung $^{\text {b }}$ and Seung Jun Chang ${ }^{c, *}$ \\ ABSTRACT. In this paper, we consider the Fourier-type functionals introduced in \\ [16]. We then establish the analytic Feynman integral for the Fourier-type func- \\ tionals. Further, we get a series expansion of the analytic Feynman integral for the \\ Fourier-type functional $\left[\Delta^{k} F\right]^{\wedge}$. We conclude by applying our series expansion to \\ several interesting functionals.
}

\section{INTRODUCTION}

Let $C_{0}[0, T]$ denote one-parameter Wiener space; that is the space of continuous real-valued functions $x$ on $[0, T]$ with $x(0)=0$. Let $\mathcal{M}$ denote the class of all Wiener measurable subsets of $C_{0}[0, T]$ and let $m$ denote Wiener measure. $\left(C_{0}[0, T], \mathcal{M}, m\right)$ is a complete measure space, and we denote the Wiener integral of a Wiener integrable functional $F$ by $\int_{C_{0}[0, T]} F(x) d m(x)$.

A subset $B$ of $C_{0}[0, T]$ is said to be scale-invariant measurable provided $\rho B$ is $\mathcal{M}$ measurable for all $\rho>0$, and a scale-invariant measurable set $N$ is said to be a scaleinvariant null set provided $m(\rho N)=0$ for all $\rho>0$. A property that holds except on a scale-invariant null set is said to hold scale-invariant almost everywhere(s-a.e.)[19]. Throughout this paper we will assume that each functional $F: C_{0}[0, T] \rightarrow \mathbb{C}$ that we consider is scale-invariant measurable and that $\int_{C_{0}[0, T]}|F(\rho x)| d m(x)<\infty$ for each $\rho>0$.

In 1948, Feynman assumed the existence of an integral over a space of paths, and he used his integral in a formal way in his approach to quantum mechanics [9]. Several mathematicians have attempted to give rigorously meaningful definitions of the Feynman integral with appropriate existence theorems and have expressed solutions

Received by the editors November 15, 2011. Revised April 18, 2012. Accepted April 25, 2012. 2000 Mathematics Subject Classification. Primary 60J65, 28C20. 42B10, 43A32.

Key words and phrases. analytic Feynman integral, Fourier transform, Fourier-type functional, hypergeometric function, Wiener space.

${ }^{*}$ Corresponding author. 
of the Schrödinger equation in terms of their integrals. One of these approaches was based on the similarity between the Wiener and the Feynman integrals, and procedures were set up by many mathematicians to obtain Feynman integrals from Wiener integrals by analytic extension from the real axis to the imaginary axis. For the procedure of analytic continuation to define the analytic Feynman integral, see [4-7].

Let $[f]^{\wedge}$ be the Fourier transform of $f$,

$$
[f]^{\wedge}(\vec{\xi})=\left(\frac{1}{2 \pi}\right)^{\frac{n}{2}} \int_{\mathbb{R}^{n}} f(\vec{u}) \exp \{i \vec{u} \cdot \vec{\xi}\} d \vec{u}, \quad \vec{u}, \vec{\xi} \in \mathbb{R}^{n},
$$

where $\vec{u} \cdot \vec{\xi}=u_{1} \xi_{1}+\cdots+u_{n} \xi_{n}$. Many mathematicians and physicists studied the Fourier transform since the Fourier transform is a very useful tool in physics and various fields including mathematics. In $[2,21]$, the authors established the PaleyWiener theorems for the Fourier transform. Their theorems were based upon some properties of the Fourier transform. In [22], Tuan established the Paley-Wiener type theorems over the appropriate domains. In [16], Chung and Tuan studied the Fourier type functionals via the Fourier transforms on Wiener space and investigated some properties of the Fourier-type functionals.

In this paper, we investigate several properties involving the analytic Feynman integral for the Fourier-type functionals $\Delta^{k} F$ and $\left[\Delta^{k} F\right]^{\wedge}$ on $C_{0}[0, T]$. In Section 3, we obtain the existence and various formulas of the analytic Feynman integral for $\Delta^{k} F$ and $\left[\Delta^{k} F\right]^{\wedge}$. Finally, in Section 4 , we present various series expansions of the analytic Feynman integral for various Fourier-type functionals.

\section{Definitions and Preliminaries}

In this section, we list some definitions and properties from [4-6, 10, 18].

For $v \in L_{2}[0, T]$ and $x \in C_{0}[0, T]$, let $\langle v, x\rangle$ denote the Paley-Wiener-Zygmund (PWZ) stochastic integral. One can show that for each $v \in L_{2}[0, T],\langle v, x\rangle$ exists for a.e. $x \in C_{0}[0, T]$ and if $v \in L_{2}[0, T]$ is a function of bounded variation on $[0, T]$, $\langle v, x\rangle$ equals the Riemann-Stieltjes integral $\int_{0}^{T} v(t) d x(t)$ for s-a.e. $x \in C_{0}[0, T]$. Also, $\langle v, x\rangle$ has the expected linearity property. Furthermore, $\langle v, x\rangle$ is a Gaussian random variable with mean 0 and variance $\|v\|_{2}^{2}$. For a more detailed study of the PWZ stochastic integral, see $[13,17,18]$.

Now, we state the definition of the analytic Feynman integral. 
Definition 2.1. Let $\mathbb{C}$ denote the complex numbers, let $\mathbb{C}_{+}=\{\lambda \in \mathbb{C}: \operatorname{Re}(\lambda)>0\}$ and let $\tilde{\mathbb{C}}_{+}=\{\lambda \in \mathbb{C}: \lambda \neq 0$ and $\operatorname{Re}(\lambda) \geq 0\}$. Let $F: C_{0}[0, T] \rightarrow \mathbb{C}$ be a measurable functional such that for each $\lambda>0$, the Wiener integral

$$
J(\lambda)=\int_{C_{0}[0, T]} F\left(\lambda^{-1 / 2} x\right) d m(x)
$$

exists. If there exists a function $J^{*}(\lambda)$ analytic in $\mathbb{C}_{+}$such that $J^{*}(\lambda)=J(\lambda)$ for all $\lambda>0$, then $J^{*}(\lambda)$ is defined to be the analytic Wiener integral of $F$ over $C_{0}[0, T]$ with parameter $\lambda$, and for $\lambda \in \mathbb{C}_{+}$we write

$$
J^{*}(\lambda)=\int_{C_{0}[0, T]}^{\mathrm{anw}_{\lambda}} F(x) d m(x) .
$$

Let $q \neq 0$ be a real number and let $F$ be a functional such that $J^{*}(\lambda)$ exists for all $\lambda \in \mathbb{C}_{+}$. If the following limit exists, we call it the analytic Feynman integral of $F$ with parameter $q$ and we write

$$
\int_{C_{0}[0, T]}^{\mathrm{anf}_{q}} F(x) d m(x)=\lim _{\lambda \rightarrow-i q} \int_{C_{0}[0, T]}^{\mathrm{anw}_{\lambda}} F(x) d m(x)
$$

where $\lambda \rightarrow-i q$ through values in $\mathbb{C}_{+}$.

The following theorem is a well-known integration formula which is used several times in this paper, see [1].

Theorem 2.2. Let $\left\{\alpha_{1}, \cdots, \alpha_{n}\right\}$ be an orthonormal set of functions from $L_{2}[0, T]$. Let $f: \mathbb{R}^{n} \rightarrow \mathbb{C}$ be Borel measurable, and let

$$
F(x)=f\left(\left\langle\alpha_{1}, x\right\rangle, \cdots,\left\langle\alpha_{n}, x\right\rangle\right) \equiv f(\langle\vec{\alpha}, x\rangle)
$$

Then

$$
\begin{aligned}
\int_{C_{0}[0, T]} F(x) d m(x) & =\int_{C_{0}[0, T]} f(\langle\vec{\alpha}, x\rangle) d m(x) \\
& =\left(\frac{1}{2 \pi}\right)^{\frac{n}{2}} \int_{\mathbb{R}^{n}} f(\vec{v}) \exp \left\{-\frac{|\vec{v}|^{2}}{2}\right\} d \vec{v}
\end{aligned}
$$

in the sense that if either side of (2.1) exists, both sides exist and equality holds.

Now we state some well-known properties of the Fourier transform as a lemma, see [22].

Lemma 2.3. (1) Let $f \in C^{\infty}\left(\mathbb{R}^{n}\right)$ be such that all Laplacians $\Delta^{k} f, k=0,1, \cdots$, belong to $L_{2}\left(\mathbb{R}^{n}\right)$. Then

$$
\left[\Delta^{k} f\right]^{\wedge}(\vec{\xi})=(-1)^{k}|\vec{\xi}|^{2 k} \hat{f}(\vec{\xi})
$$


where

$$
|\vec{\xi}|=\sqrt{\xi_{1}^{2}+\cdots+\xi_{n}^{2}} \quad \text { and } \quad \Delta=\frac{\partial^{2}}{\partial \xi_{1}^{2}}+\cdots+\frac{\partial^{2}}{\partial \xi_{n}^{2}} .
$$

(2) The following statement is called the Paley-Wiener-type theorem for the Fourier transform on $\mathbb{R}^{n}$. A function $f$, square integrable on $\mathbb{R}^{n}$, is the restriction on $\mathbb{R}^{n}$ of an entire function of exponential type if, and only if $\Delta^{k} f$ belong to $L_{2}\left(\mathbb{R}^{n}\right)$ for all positive integers $k$.

Let $\mathcal{S}\left(\mathbb{R}^{n}\right)$ be the Schwartz space of infinitely differentiable function $f(\vec{u})$ decaying at infinity together with all its derivatives faster than any polynomial of $|\vec{u}|^{-1}$. Note that the Fourier transform is an isomorphism on the Schwartz space $\mathcal{S}\left(\mathbb{R}^{n}\right)$. Also, $\Delta^{k} f$ and $\left[\Delta^{k} f\right]^{\wedge}$ are elements on $\mathcal{S}\left(\mathbb{R}^{n}\right)$ for all $k=1,2, \cdots$.

Now we introduce the Fourier-type functionals defined on $C_{0}[0, T]$.

Definition 2.4. Let $\left\{\alpha_{1}, \cdots, \alpha_{n}\right\}$ be an orthonormal set of functions from $L_{2}[0, T]$. For $f \in \mathcal{S}\left(\mathbb{R}^{n}\right)$, let

$$
\Delta^{k} F(x)=\left(\Delta^{k} f\right)(\langle\vec{\alpha}, x\rangle), \quad k=0,1, \cdots
$$

and

$$
\left[\Delta^{k} F\right]^{\wedge}(x)=\left[\Delta^{k} f\right]^{\wedge}(\langle\vec{\alpha}, x\rangle), \quad k=0,1, \cdots .
$$

The functionals in (2.3) and (2.4) are called the Fourier-type functionals defined on Wiener space $C_{0}[0, T]$.

Remark 2.5. (1) The Fourier-type functionals appear frequently in the applications of the analytic Feynman integral to quantum theory, see Section 4.

(2) Using equation (2.2), the Fourier-type functional $\left[\Delta^{k} F\right]^{\wedge}$ given by equation (2.4) can be expressed by

$$
\left[\Delta^{k} F\right]^{\wedge}(x)=(-1)^{k}|\langle\vec{\alpha}, x\rangle|^{2 k} \hat{f}(\langle\vec{\alpha}, x\rangle)=(-1)^{k}|\langle\vec{\alpha}, x\rangle|^{2 k}[F]^{\wedge}(x) .
$$

\section{Analytic Feynman Integral for the Fourier-type FUNCTIONALS}

In this section we establish the analytic Feynman integral for the Fourier-type functionals given by equations (2.3) and (2.4). We then establish some properties of the analytic Feynman integrals for $\Delta^{k} F$ and $\left[\Delta^{k} F\right]^{\wedge}$ and their relationships.

In our next two theorems, we obtain the analytic Feynman integral for the Fourier-type functionals for $\Delta^{k} F$ and $\left[\Delta^{k} F\right]^{\wedge}$, respectively. 
Theorem 3.1. Let $\Delta^{k} F$ be given by equation (2.3). Then for all $q \in \mathbb{R}-\{0\}$, the analytic Feynman integral of $\Delta^{k} F$ exists and is given by the formula

$$
\int_{C_{0}[0, T]}^{\operatorname{anf}_{q}} \Delta^{k} F(x) d m(x)=\left(\frac{-i q}{2 \pi}\right)^{\frac{n}{2}} \int_{\mathbb{R}^{n}}\left(\Delta^{k} f\right)(\vec{v}) \exp \left\{\frac{i q|\vec{v}|^{2}}{2}\right\} d \vec{v} .
$$

Proof. By using equation (2.1), we have for all $\lambda>0$

$$
\int_{C_{0}[0, T]} \Delta^{k} F\left(\lambda^{-1 / 2} x\right) d m(x)=\left(\frac{\lambda}{2 \pi}\right)^{\frac{n}{2}} \int_{\mathbb{R}^{n}}\left(\Delta^{k} f\right)(\vec{v}) \exp \left\{-\frac{\lambda|\vec{v}|^{2}}{2}\right\} d \vec{v} .
$$

By using Morera's theorem, the right-side term in the above equality is an analytic function of $\lambda \in \mathbb{C}_{+}$and is a continuous function of $\lambda \in \tilde{\mathbb{C}}_{+}$and so setting $\lambda=-i q$ yields equation (3.1).

Next, we prove the existence theorem of analytic Feynman integral. Since $\Delta^{k} f \in$ $\mathcal{S}\left(\mathbb{R}^{n}\right)$, using equation (3.1),

$$
\left|\int_{C_{0}[0, T]}^{\operatorname{anf}_{q}} \Delta^{k} F(x) d m(x)\right| \leq\left(\frac{|q|}{2 \pi}\right)^{\frac{n}{2}} \int_{\mathbb{R}^{n}}\left|\left(\Delta^{k} f\right)(\vec{v})\right| d \vec{v}<\infty .
$$

Thus we have the desired result.

The following Theorem 3.2 is the main result in this section. This theorem gives us the existence for the series expansion of the analytic Feynman integral for $\left[\Delta^{k} F\right]^{\wedge}$, see Theorem 4.1 below.

Theorem 3.2. Let $\left[\Delta^{k} F\right]^{\wedge}$ be given by equation (2.4). Then for all $q \in \mathbb{R}-\{0\}$, the analytic Feynman integral of $\left[\Delta^{k} F\right]^{\wedge}$ exists and is given by the formula

$$
\int_{C_{0}[0, T]}^{\operatorname{anf}_{q}}\left[\Delta^{k} F\right]^{\wedge}(x) d m(x)=\left(\frac{1}{2 \pi}\right)^{\frac{n}{2}} \int_{\mathbb{R}^{n}}\left(\Delta^{k} f\right)(\vec{v}) \exp \left\{-\frac{i|\vec{v}|^{2}}{2 q}\right\} d \vec{v} .
$$

Proof. By using equations (2.1) and (1.1), we have for all $\lambda>0$

$$
\begin{aligned}
& \int_{C_{0}[0, T]}\left[\Delta^{k} F\right]^{(}\left(\lambda^{-1 / 2} x\right) d m(x)=\left(\frac{\lambda}{2 \pi}\right)^{\frac{n}{2}} \int_{\mathbb{R}^{n}}\left[\Delta^{k} f \widehat{\Upsilon}^{\wedge}(\vec{\xi}) \exp \left\{-\frac{\lambda|\vec{\xi}|^{2}}{2}\right\} d \vec{\xi}\right. \\
& =\left(\frac{\lambda}{2 \pi}\right)^{\frac{n}{2}}\left(\frac{1}{2 \pi}\right)^{\frac{n}{2}} \int_{\mathbb{R}^{n}}\left(\Delta^{k} f\right)(\vec{v}) \int_{\mathbb{R}^{n}} \exp \left\{-\frac{\lambda|\vec{\xi}|^{2}}{2}+i \vec{v} \cdot \vec{\xi}\right\} d \vec{\xi} d \vec{v} \\
& =\left(\frac{1}{2 \pi}\right)^{\frac{n}{2}} \int_{\mathbb{R}^{n}}\left(\Delta^{k} f\right)(\vec{v}) \exp \left\{-\frac{|\vec{v}|^{2}}{2 \lambda}\right\} d \vec{v} .
\end{aligned}
$$

By using Morera's theorem, the last expression above is an analytic function of $\lambda \in \mathbb{C}_{+}$and is a continuous function of $\lambda \in \tilde{\mathbb{C}}_{+}$and so setting $\lambda=-i q$ yields equation (3.3). Finally, using the similar method of equation (3.2), we can easily 
obtain the existence of the analytic Feynman integral. Thus we have the desired result.

Many mathematicians use the properties for the convolution of the Fourier transform to solve appropriate differential equations in their research fields. In Theorems 3.1 and 3.2 we considered the Fourier-type functional via the Fourier transform. So we need a new concept which looks like convolution of the Fourier transform.

Next we give the definition of $\diamond$-convolution of functionals on $C_{0}[0, T]$.

Definition 3.3. Let $F$ be as in Definition 2.4 and let $G$ be the Fourier-type functional on $C_{0}[0, T]$ of the form

$$
G(x)=g(\langle\vec{\alpha}, x\rangle) .
$$

We define their $\diamond$-convolution by

$$
(F \diamond G)(x)=(f * g)(\langle\vec{\alpha}, x\rangle) .
$$

Remark 3.4. The $\diamond$-convolution preserves useful properties of convolution for the Fourier transform as follows. Let $F$ and $G$ be the Fourier-type functionals on $C_{0}[0, T]$.

$$
(F \diamond G)=(G \diamond F), \quad[F]^{\wedge} \diamond[G]^{\wedge}=(2 \pi)^{\frac{n}{2}}[F G]^{\wedge}
$$

and

$$
[(F \diamond G)]^{\wedge}=(2 \pi)^{\frac{n}{2}}[F]^{\widehat{[}}[G]^{\wedge}=[(G \diamond F)]^{\hat{\prime}} ;
$$

that is to say the $\diamond$-convolution is commutative.

In our next theorem, we establish the Feynman integration formulas (3.4)-(3.7) by using Remark 3.4 and equation (2.5).

Theorem 3.5. Let $F$ and $G$ be as in Remark 3.4. Then for all $q \in \mathbb{R}-\{0\}$,

$$
\begin{gathered}
\int_{C_{0}[0, T]}^{\operatorname{anf}_{q}}\left[(F \diamond G) \hat{]^{\prime}}(x) d m(x)=(2 \pi)^{\frac{n}{2}} \int_{C_{0}[0, T]}^{\operatorname{anf}_{q}}[F]^{\wedge}(x)[G]^{\wedge}(x) d m(x),\right. \\
\int_{C_{0}[0, T]}^{\operatorname{anf}_{q}}\left(\Delta^{k} F \diamond G\right)(x) d m(x)=\int_{C_{0}[0, T]}^{\operatorname{anf}_{q}}\left(F \diamond \Delta^{k} G\right)(x) d m(x), \\
\int_{C_{0}[0, T]}^{\operatorname{anf}_{q}}\left[\left(\Delta^{k} F \diamond G\right)\right]^{\wedge}(x) d m(x)=(2 \pi)^{\frac{n}{2}} \int_{C_{0}[0, T]}^{\operatorname{anf}_{q}}\left([F]^{\wedge}(x)\left[\Delta^{k} G\right]^{\jmath}\right)(x) d m(x), \\
\int_{C_{0}[0, T]}^{\operatorname{anf}_{q}}\left[\Delta^{k} F\right]^{\wedge}(x) \diamond\left[\Delta^{k} G\right]^{\wedge}(x) d m(x)=(2 \pi)^{\frac{n}{2}} \int_{C_{0}[0, T]}^{\operatorname{anf}_{q}}\left[\Delta^{k} F \Delta^{k} G\right]^{\wedge}(x) d m(x)
\end{gathered}
$$


and

$$
\int_{C_{0}[0, T]}^{\operatorname{anf}_{q}}\left[\Delta^{k} F \diamond \Delta^{k} G\right]^{\wedge}(x) d m(x)=(2 \pi)^{\frac{n}{2}} \int_{C_{0}[0, T]}^{\operatorname{anf}_{q}}|\langle\vec{\alpha}, x\rangle|^{4 k}[F]^{\wedge}(x)[G]^{\wedge}(x) d m(x) .
$$

In particular, if $k$ is even, then

$$
\int_{C_{0}[0, T]}^{\operatorname{anf}_{q}}\left[\Delta^{k / 2} F \diamond \Delta^{k / 2} F\right]^{\wedge}(x) d m(x)=(2 \pi)^{\frac{n}{2}} \int_{C_{0}[0, T]}^{\operatorname{anf}_{q}}\left(\left[\Delta^{k / 2} F\right]^{`}\right)^{2}(x) d m(x)
$$

and

$$
\int_{C_{0}[0, T]}^{\operatorname{anf}_{q}}\left[\Delta^{k / 2} F\right]^{\wedge}(x) \diamond\left[\Delta^{k / 2} F\right]^{\wedge}(x) d m(x)=(2 \pi)^{\frac{n}{2}} \int_{C_{0}[0, T]}^{\operatorname{anf}_{q}}\left[\left(\Delta^{k / 2} F\right)^{2}\right]^{\wedge}(x) d m(x) .
$$

\section{Series Expansions of the Analytic Feynman Integral FOR THE FOURIER-TYPE FunCTIONALS}

In this section, we will investigate a series representation of the the analytic Feynman integral for the Fourier-type functional using hypergeometric series expansion which is different form Taylor series expansion. In addition we explain the Fouriertype functional applied to several areas. We then give the special applications for each case.

The following statement is certainly known and the result plays a key role in this section. The hypergeometric function has a hypergeometric series given by

$$
\begin{aligned}
{ }_{1} \mathcal{F}_{1}[a, b, z]= & 1+\frac{a}{b} z+\frac{a(a+1)}{b(b+1)} \frac{z^{2}}{2 !}+\frac{a(a+1)(a+2)}{b(b+1)(b+2)} \frac{z^{3}}{3 !} \\
& +\frac{a(a+1)(a+2)(a+3)}{b(b+1)(b+2)(b+3)} \frac{z^{4}}{4 !}+\cdots=\sum_{s=0}^{\infty} \frac{(a)_{s}}{(b)_{s}} \frac{z^{s}}{s !}
\end{aligned}
$$

where $(a)_{s}$ is the Pochhammer symbol; that is to say

$$
(a)_{s}=a(a+1)(a+2) \cdots(a+s-1) .
$$

If $a$ and $b$ are integers, $a<0$ and either $b>0$ or $b<a$, then the series yields a polynomial with a finite number of terms. In particular, $(a)_{0}=1$. When $b \in$ $\mathbb{Z}^{-} \cup\{0\},{ }_{1} \mathcal{F}_{1}[a, b, z]$ is undefined.

In our next theorem we obtain the series expansion of the analytic Feynman integral for $\left[\Delta^{k} F\right]^{\wedge}$. Equation (4.2) is the series representation of the the analytic Feynman integral for the Fourier-type functional $\left[\Delta^{k} F\right]^{\wedge}$ using hypergeometric series expansion. 
Theorem 4.1. Let $\left[\Delta^{k} F\right]^{\wedge}$ be given by equation (2.4). Then the analytic Feynman integral for $\left[\Delta^{k} F\right]^{\wedge}$ exists and for all $q \in \mathbb{R}-\{0\}$,

$$
\begin{aligned}
& \int_{C_{0}[0, T]}^{\operatorname{anf}_{q}}\left[\Delta^{k} F\right]^{\wedge}(x) d m(x) \\
& =\left(\frac{\sqrt{-i q}}{2 \pi}\right)^{n} \sum_{l_{1}+\cdots+l_{n}=k} \frac{(-1)^{k} k !}{l_{1} ! \cdots l_{n} !} \int_{\mathbb{R}^{n}} f(\vec{u}) \exp \left\{-\frac{i|\vec{u}|^{2}}{2 q}\right\} \\
& \cdot \prod_{j=1}^{n}\left(\frac{2 i}{q}\right)^{l_{j}} \frac{2 \Gamma\left(\frac{1}{2}+l_{j}\right)}{\sqrt{-2 i q}}{ }_{1} \mathcal{F}_{1}\left[-l_{j}, \frac{1}{2}, \frac{i u_{j}^{2}}{2 q}\right] d \vec{u}
\end{aligned}
$$

where ${ }_{1} \mathcal{F}_{1}$ is given by equation (4.1) and $\Gamma$ is the Gamma function.

Proof. First note that using Theorem 3.2, the analytic Feynman integral for $\left[\Delta^{k} F\right]^{-}$ always exists. Now we will obtain the series expansion of the analytic Feynman integral for $\left[\Delta^{k} F\right]^{\wedge}$. By using equations (2.5) and (1.1), we have for all $\lambda>0$

$$
\begin{gathered}
\int_{C_{0}[0, T]}\left[\Delta^{k} F\right]^{\wedge}\left(\lambda^{-1 / 2} x\right) d m(x)=\left(\frac{\lambda}{2 \pi}\right)^{\frac{n}{2}}(-1)^{k} \int_{\mathbb{R}^{n}}|\vec{\xi}|^{2 k} \hat{f}(\vec{\xi}) \exp \left\{-\frac{\lambda|\vec{\xi}|^{2}}{2}\right\} d \vec{\xi} \\
=\left(\frac{\lambda}{2 \pi}\right)^{\frac{n}{2}}(-1)^{k}\left(\frac{1}{2 \pi}\right)^{\frac{n}{2}} \int_{\mathbb{R}^{n}}|\vec{\xi}|^{2 k} \int_{\mathbb{R}^{n}} f(\vec{u}) \exp \{i \vec{u} \cdot \vec{\xi}\} d \vec{u} \exp \left\{-\frac{\lambda|\vec{\xi}|^{2}}{2}\right\} d \vec{\xi} \\
=\left(\frac{\lambda}{2 \pi}\right)^{\frac{n}{2}}(-1)^{k}\left(\frac{1}{2 \pi}\right)^{\frac{n}{2}} \int_{\mathbb{R}^{n}} f(\vec{u}) \exp \left\{-\frac{|\vec{u}|^{2}}{2 \lambda}\right\} \\
\cdot\left[\int_{\mathbb{R}^{n}}|\vec{\xi}|^{2 k} \exp \left\{-\frac{\lambda}{2}\left|\vec{\xi}-\frac{i \vec{u}}{\lambda}\right|^{2}\right\} d \vec{\xi}\right] d \vec{u} .
\end{gathered}
$$

Now by using the multinomial theorem, the last equation above equals

$$
\begin{aligned}
&\left(\frac{\lambda}{2 \pi}\right)^{\frac{n}{2}}(-1)^{k}\left(\frac{1}{2 \pi}\right)^{\frac{n}{2}} \int_{\mathbb{R}^{n}} f(\vec{u}) \exp \left\{-\frac{|\vec{u}|^{2}}{2 \lambda}\right\} \\
& \cdot\left[\int_{\mathbb{R}^{n}} \sum_{l_{1}+\cdots+l_{n}=k} \frac{k !}{l_{1} ! \cdots l_{n} !} \xi_{1}^{2 l_{1}} \xi_{2}^{2 l_{2}} \cdots \xi_{n}^{2 l_{n}} \exp \left\{-\frac{\lambda}{2}\left|\vec{\xi}-\frac{i \vec{u}}{\lambda}\right|^{2}\right\} d \vec{\xi}\right] d \vec{u}
\end{aligned}
$$

Finally, by using the Mathematica program we have the following formula

$$
\int_{\mathbb{R}} \xi^{2 k} \exp \left\{-\frac{\lambda}{2}\left(\xi-\frac{i u}{\lambda}\right)^{2}\right\} d \xi=\left(\frac{2}{\lambda}\right)^{k} \frac{2 \Gamma\left(\frac{1}{2}+k\right)}{\sqrt{2} \sqrt{\lambda}}{ }_{1} \mathcal{F}_{1}\left[-k, \frac{1}{2}, \frac{u^{2}}{2 \lambda}\right],
$$

where $\Gamma$ is the Gamma function. Applying this to the last equation above, we get 


$$
\begin{aligned}
& \int_{C_{0}[0, T]}\left[\Delta^{k} F\right]^{\wedge}\left(\lambda^{-1 / 2} x\right) d m(x) \\
& =\left(\frac{\lambda}{2 \pi}\right)^{\frac{n}{2}}(-1)^{k}\left(\frac{1}{2 \pi}\right)^{\frac{n}{2}} \int_{\mathbb{R}^{n}} f(\vec{u}) \exp \left\{-\frac{|\vec{u}|^{2}}{2 \lambda}\right\} \\
& \cdot\left[\sum_{l_{1}+\cdots+l_{n}=k} \frac{k !}{l_{1} ! \cdots l_{n} !} \prod_{j=1}^{n}\left(\frac{2}{\lambda}\right)^{l_{j}} \frac{2 \Gamma\left(\frac{1}{2}+l_{j}\right)}{\sqrt{2 \lambda}}{ }_{1} \mathcal{F}_{1}\left[-l_{j}, \frac{1}{2}, \frac{u_{j}^{2}}{2 \lambda}\right]\right] d \vec{u} .
\end{aligned}
$$

By using Morera's theorem, the last expression above is an analytic function of $\lambda \in \mathbb{C}_{+}$and is a continuous function of $\lambda \in \tilde{\mathbb{C}}_{+}$and so setting $\lambda=-i q$ yields equation (4.2). Thus we have the desired result.

Now we exhibit several Fourier-type functionals to apply Theorem 4.1 above. We then compute the analytic Feynman integrals for special cases by using the series expansion for each Fourier-type functionals.

1. The heat equation in one dimension. Consider the heat equation in one dimension

$$
\left\{\begin{array}{l}
u_{t}=u_{y y} \\
u(y, 0)=\phi(y)
\end{array} .\right.
$$

Take the Fourier transform of both sides with respect to $y$,

$$
\left[u_{t}\right]^{\wedge}=\left[u_{y y}\right]^{\wedge}=-\xi^{2}[u]^{\wedge} \text { for } \xi \in \mathbb{R} \text {. }
$$

Solve the differential equation $\left[u_{t}\right]^{\wedge}=-\xi^{2}[u]^{\wedge}$ to get

$$
[u]^{\wedge}(\xi, t)=C(\xi) \exp \left\{-\xi^{2} t\right\} \text {. }
$$

By using the initial condition, $[u]^{\wedge}(\xi, t)=\hat{\phi}(\xi) \exp \left\{-\xi^{2} t\right\}$. Now using the formula for the Fourier transform,

$$
\exp \left\{-\xi^{2} t\right\}=\frac{1}{\sqrt{2 t}}\left[\exp \left\{-y^{2} / 4 t\right\}\right]
$$

Let

$$
s_{t}(y)=\frac{1}{\sqrt{2 t}} \exp \left\{-y^{2} / 4 t\right\}
$$

Then $[u]^{\wedge}=[\phi]^{\wedge}\left[s_{t}\right]^{\wedge}=\left[\phi * s_{t}\right]^{\wedge}$. Thus the solution of the heat equation is $\phi * s_{t}$. The function $s_{t}$ is an element of the Schwartz class $\mathcal{S}(\mathbb{R})$. 
Let $\Delta^{k} S_{t}(x)=\Delta^{k} s_{t}\left(\left\langle\alpha_{1}, x\right\rangle\right)$. Then, using Theorem 4.1, the analytic Feynman integral of $\left[\Delta^{k} S_{t}\right]^{\wedge}$ exists and using equation (4.2), we have

$$
\begin{aligned}
& \int_{C_{0}[0, T]}^{\operatorname{anf}_{q}}\left[\Delta^{k} S_{t}\right]^{\wedge}(x) d m(x) \\
& =\frac{(-1)^{k}}{2 \pi \sqrt{t}}\left(\frac{2 i}{q}\right)^{k} \Gamma\left(\frac{1}{2}+k\right) \int_{\mathbb{R}} \exp \left\{-\frac{u^{2}}{4 t}-\frac{i u^{2}}{2 q}\right\}_{1} \mathcal{F}_{1}\left[-k, \frac{1}{2}, \frac{i u^{2}}{2 q}\right] d u .
\end{aligned}
$$

For examples, we compute the analytic Feynman integrals of the Fourier-type functional $\left[\Delta^{k} S_{t}\right]^{\wedge}$ as follows:

\begin{tabular}{l|l}
\hline & $\int_{C_{0}[0, T]}^{\operatorname{anf}_{q}\left[\Delta^{k} S_{t}\right]^{\wedge}(x) d m(x), \quad t>0}$ \\
\hline$k=1$ & $\frac{i}{8 q^{2} \sqrt{t}}\left(\frac{1}{4 t}+\frac{i}{2 q}\right)^{-\frac{5}{2}}\left(\frac{-2 i-q}{4 t^{2}}\right)$ \\
\hline$k=2$ & $\frac{-3}{32 q^{2} t^{2} \sqrt{t}}\left(\frac{1}{4 t}+\frac{i}{2 q}\right)^{-\frac{5}{2}}$ \\
\hline$k=3$ & $\frac{15 i}{q^{3}(q+2 i t)^{3} \sqrt{t}}\left(\frac{1}{4 t}+\frac{i}{2 q}\right)^{-\frac{1}{2}}\left(16 t^{3} i+12 q t^{2}\right)$ \\
\hline
\end{tabular}

Table 1. Heat equation in one dimension

2. The Hermite function. For $n=0,1, \cdots$, define the Hermite function by

$$
p_{n}(u)=\frac{1}{\sqrt{2^{n} n ! \sqrt{\pi}}} \exp \left\{-\frac{u^{2}}{2}\right\} h_{n}(u)
$$

where

$$
h_{n}(u)=(-1)^{n} \exp \left\{u^{2}\right\} \frac{d^{n}}{d u^{n}} \exp \left\{-u^{2}\right\}
$$

is a Hermite polynomial. Next let $\left\{\beta_{p}(t): p=1,2, \cdots\right\}$ be a complete orthonormal set of functions of bounded variation on $[0, T]$. Define

$$
\Phi_{n, \rho}(x)=p_{n}\left(\int_{0}^{T} \beta_{\rho}(t) d x(t)\right), n=0,1,2, \cdots, \rho=1,2, \cdots
$$

and

$$
\Psi_{n_{1}, \cdots, n_{\rho}}(x)=\Psi_{n_{1}, \cdots, n_{\rho}, 0, \cdots, 0}(x)=\Phi_{n_{1}, 1}(x) \cdots \Phi_{n_{\rho}, \rho}(x) .
$$

The functionals in (4.5) are called the Fourier-Hermite functionals. In [3], Cameron and Martin showed that the Fourier-Hermite functionals form a complete orthonormal set in $L_{2}\left(C_{0}[0, T]\right)$. That is to say that every functional $F \in L_{2}\left(C_{0}[0, T]\right)$ has a 
Fourier-Hermite development which converges in the $L_{2}\left(C_{0}[0, T]\right)$ sense to $F$; namely that

$$
F(y)=\text { l.i.m. } \cdot_{N \rightarrow \infty} F_{N}(y)
$$

where

$$
F_{N}(y)=\sum_{n_{1}, \cdots, n_{N}=0}^{N} A_{n_{1}, \cdots, n_{N}}^{F} \Psi_{n_{1}, \cdots, n_{N}}(y)
$$

and where $A_{n_{1}, \cdots, n_{N}}^{F}$ is the Fourier-Hermite coefficient

$$
A_{n_{1}, \cdots, n_{N}}^{F}=\int_{C_{0}[0, T]} F(y) \Psi_{n_{1}, \cdots, n_{N}}(y) d m(y) .
$$

In $[11,12]$, the authors extended the results $[3,4]$ to a very general function space $C_{a, b}[0, T]$ rather than the Wiener space $C_{0}[0, T]$. The Hermite function $p_{n}$ is an element of Schwartz class $\mathcal{S}(\mathbb{R})$.

Let $\Delta^{k} P_{n}(x)=\Delta^{k} p_{n}\left(\left\langle\alpha_{1}, x\right\rangle\right)$. Then, using Theorem 4.1, the analytic Feynman integral of $\left[\Delta^{k} P_{n}\right]^{\wedge}$ exists and using equation (4.2), we have

$$
\begin{aligned}
& \int_{C_{0}[0, T]}^{\operatorname{anf}_{q}}\left[\Delta^{k} P_{n}\right]^{\wedge}(x) d m(x) \\
& =\frac{(-1)^{k}}{\pi \sqrt{2^{n} n ! \sqrt{\pi}}}\left(\frac{2 i}{q}\right)^{k} \Gamma\left(\frac{1}{2}+k\right) \int_{\mathbb{R}} \exp \left\{-\frac{u^{2}}{2}-\frac{i u^{2}}{2 q}\right\} h_{n}(u)_{1} \mathcal{F}_{1}\left[-k, \frac{1}{2}, \frac{i u^{2}}{2 q}\right] d u .
\end{aligned}
$$

For examples, we compute the analytic Feynman integrals of the Fourier-type functional $\left[\Delta^{k} P_{n}\right]^{\wedge}$ as follows:

\begin{tabular}{l|l}
\hline & $\int_{C_{0}[0, T]}^{\operatorname{anf}_{q}}\left[\Delta^{k} P_{n}\right]^{\hat{N}}(x) d m(x)$ \\
\hline$k=1(n=1)$ & $\frac{-5-i q}{8 q^{2} \sqrt{\sqrt{\pi}}}\left(\frac{1}{2}+\frac{i}{2 q}\right)^{-\frac{5}{2}}$ \\
\hline$k=2(n=2)$ & $\frac{-3 \pi}{16 q^{4} \sqrt{\pi \sqrt{\pi}}}\left(\frac{1}{2}+\frac{i}{2 q}\right)^{-\frac{5}{2}}\left(12 i q-3-\frac{i}{q}-\frac{(i+q)^{3}}{q}\right)$ \\
\hline$k=3(n=2)$ & $\frac{15 \sqrt{2} i}{q^{4} \sqrt{3 \sqrt{\pi}}\left(1+\frac{i}{q}\right)^{-\frac{9}{2}}}$ \\
\hline
\end{tabular}

Table 2. The Hermite function 
3. The Gauss kernel. For $\varepsilon>0$ and $\vec{u} \in \mathbb{R}^{n}$, the Gauss kernel is given by

$$
g_{\varepsilon}(\vec{u})=\left(\frac{1}{4 \pi \varepsilon}\right)^{\frac{n}{2}} \exp \left\{-\frac{|\vec{u}|^{2}}{4 \varepsilon}\right\} .
$$

Then the Gauss kernel $g_{\varepsilon}$ has some useful properties:

(1) $g_{\varepsilon}(\vec{u}) \geq 0$ for all $u$,

(2) $\int_{\mathbb{R}^{n}} g_{\varepsilon}(\vec{u}) d \vec{u}=1$ for all $\varepsilon>0$

and

(3) For $\delta>0, \lim _{\varepsilon \rightarrow 0} \int_{|\vec{u}| \geq \delta} g_{\varepsilon}(\vec{u}) d \vec{u}=0$.

Furthermore, we can show that the Gauss kernel $g_{\varepsilon}(\vec{u})$ from (1) thru (3) above,

$$
\lim _{\varepsilon \rightarrow 0} \int g_{\varepsilon}(\vec{u}-\vec{v}) f(\vec{v}) d \vec{v}=f(\vec{u})
$$

for all $f \in \mathcal{S}\left(\mathbb{R}^{n}\right)$. The $g_{\varepsilon}$ is called an approximate identity as $\varepsilon \rightarrow 0$. Also, the Gauss kernel is an element of Schwartz class $\mathcal{S}\left(\mathbb{R}^{n}\right)$.

Let $\Delta^{k} G_{\varepsilon}(x)=\Delta^{k} g_{\varepsilon}(\langle\vec{\alpha}, x\rangle)$. Then, using Theorem 4.1, the analytic Feynman integral of $\left[\Delta^{k} G_{\varepsilon}\right]^{\wedge}$ exists and using equation (4.2), we have

$$
\begin{aligned}
& \int_{C_{0}[0, T]}^{\operatorname{anf}_{q}}\left[\Delta^{k} G_{\varepsilon}\right]^{\wedge}(x) d m(x)=\left(\frac{\sqrt{-i q}}{2 \pi}\right)^{n} \sum_{l_{1}+\cdots+l_{n}=k} \frac{(-1)^{k} k !}{l_{1} ! \cdots l_{n} !} \int_{\mathbb{R}^{n}}\left(\frac{1}{4 \pi \varepsilon}\right)^{\frac{n}{2}} \\
& \cdot \exp \left\{-\frac{|\vec{u}|^{2}}{4 \varepsilon}-\frac{i|\vec{u}|^{2}}{2 q}\right\} \prod_{j=1}^{n}\left(\frac{2 i}{q}\right)^{l_{j}} \frac{2 \Gamma\left(\frac{1}{2}+l_{j}\right)}{\sqrt{-2 i q}}{ }_{1} \mathcal{F}_{1}\left[-l_{j}, \frac{1}{2}, \frac{i u_{j}^{2}}{2 q}\right] d \vec{u} .
\end{aligned}
$$

For examples, we compute the analytic Feynman integrals of the Fourier-type functional $\left[\Delta^{k} G_{\varepsilon}\right]^{\wedge}$ as follows:

\begin{tabular}{l|l}
\hline & $\int_{C_{0}[0, T]}^{\operatorname{anf}_{q}}\left[\Delta^{k} G_{\varepsilon}\right]^{-}(x) d m(x), \quad \varepsilon>0$ \\
\hline$k=1(n=2)$ & $\frac{-i}{32 \pi q^{2} \varepsilon}\left(\frac{1}{4 \varepsilon}+\frac{i}{2 q}\right)^{-3}\left(-\frac{i}{\varepsilon}-\frac{q}{2 \varepsilon^{2}}\right)$ \\
\hline$k=2(n=2)$ & $\frac{-1}{64 \pi q^{4} \varepsilon}\left(\frac{1}{4 \varepsilon}+\frac{i}{2 q}\right)^{-5}\left(-\frac{i}{2 \varepsilon}-\frac{q}{4 \varepsilon^{2}}\right)^{2}$ \\
& $-\frac{3 \sqrt{-i q}}{64 \pi^{2} q^{2} \varepsilon^{3} \sqrt{2}}\left(\frac{1}{4 \varepsilon}+\frac{i}{2 q}\right)^{-\frac{5}{2}}$ \\
\hline$k=3(n=2)$ & $\frac{15 i \sqrt{\pi}}{8 \pi^{2} q^{2} \varepsilon}\left(\frac{1}{4 \pi \varepsilon}\right)^{\frac{3}{2}}\left(\frac{1}{4 \varepsilon}+\frac{i}{2 q}\right)^{-1}$ \\
& $\left(-4 i \varepsilon^{3}-12 \varepsilon^{2} q+24 \varepsilon^{3} i q+12 \varepsilon^{2} q^{2}-q^{3}\right)$ \\
& $-\frac{3 i(\varepsilon i+q)}{16 \pi^{3} q^{8} \varepsilon^{5} \sqrt{\pi \varepsilon}}\left(\frac{1}{4 \varepsilon}+\frac{i}{2 q}\right)^{-4}$ \\
\hline
\end{tabular}

Table 3. The Gauss kernel 
4. Potential function in a Schrödinger equation. For $a \in \mathbb{R}$, consider the differential equation

$$
\frac{\partial}{\partial t} \psi(x, t)=\frac{1}{2 \lambda} \Delta \psi(x, t)-\frac{a^{2}}{2 \lambda} x^{2} \psi(x, t)
$$

with the initial condition $\psi(x, 0)=\varphi(x)$. For $\lambda>0$, this is the diffusion equation with the potential $V(x)=\frac{a^{2} x^{2}}{2 \lambda}$. From the Feynman-Kac formula we know that the solution of this equation can be written as a Wiener integral

$$
\int_{C_{0}[0, T]} \varphi\left(\lambda^{-1 / 2} x(T)\right) \exp \left\{-\frac{a^{2}}{2 \lambda^{2}} \int_{0}^{T} x^{2}(s) d s\right\} d m(x) .
$$

Let

$$
C_{0}^{\prime}[0, T]=\left\{w \in C_{0}[0, T]: w(t)=\int_{0}^{t} w(s) d s, w \in L_{2}[0, T]\right\} .
$$

Then it is a separable infinite dimensional Hilbert space with inner product

$$
\left(w_{1}, w_{2}\right)_{c_{0}^{\prime}}=\int_{0}^{T} w_{1}^{\prime}(t) w_{2}^{\prime}(t) d t .
$$

As is known, $\left(C_{0}^{\prime}[0, T], C_{0}[0, T], m\right)$ is an example of abstract Wiener space [20].

Let $S: C_{0}^{\prime}[0, T] \rightarrow C_{0}^{\prime}[0, T]$ be the linear operator defined by

$$
S w(t)=\int_{0}^{t} w(s) d s
$$

Then we see that the adjoint operator $S^{*}$ of $S$ is given by

$$
S^{*} w(t)=w(T) t-\int_{0}^{t} w(s) d s=\int_{0}^{t}[w(T)-w(s)] d s
$$

and the linear operator $A=S^{*} S$ is given by

$$
A w(t)=\int_{0}^{T} \min \{s, t\} w(s) d s .
$$

Furthermore, we see that $A$ is a self-adjoint operator on $C_{0}^{\prime}[0, T]$ and that

$$
\left(w_{1}, A w_{2}\right)_{c_{0}^{\prime}}=\left(S w_{1}, S w_{2}\right)_{c_{0}^{\prime}}=\int_{0}^{T} w_{1}(s) w_{2}(s) d s
$$

for all $w_{1}, w_{2} \in C_{0}^{\prime}[0, T]$. Hence $A$ is a positive definite operator, i.e., $(w, A w)_{c_{0}^{\prime}} \geq 0$ for all $w \in C_{0}^{\prime}[0, T]$.

One can show that the orthonormal eigenfunctions $\left\{\alpha_{m}\right\}$ of $A$ are given by

$$
\alpha_{m}(t)=\frac{\sqrt{2 T}}{\left(m-\frac{1}{2}\right) \pi} \sin \left(\frac{\left(m-\frac{1}{2}\right) \pi}{T} t\right)
$$


with corresponding eigenvalues $\left\{\beta_{m}\right\}$ given by

$$
\beta_{m}=\left(\frac{T}{\left(m-\frac{1}{2}\right) \pi}\right)^{2} .
$$

Furthermore, it can be shown that $\left\{\alpha_{m}\right\}$ is a basis of $C_{0}^{\prime}[0, T]$ and that $A$ is a trace class operator and so $S$ is a Hilbert-Schmidt operator on $C_{0}^{\prime}[0, T]$. In fact, the trace of $A$ is given by $\operatorname{Tr} A=\frac{1}{2} T^{2}=\int_{0}^{T} t d t$.

In this case,

$$
\int_{0}^{T} x^{2}(s) d s=\lim _{m \rightarrow \infty} \sum_{j=1}^{m} \beta_{j}\left\langle\alpha_{j}, x\right\rangle^{2}
$$

for a.e. $x \in C_{0}[0, T]$, see $[14,15]$. In $[10]$, the authors extended this result to the function space $C_{a, b}[0, T]$.

Let $f_{m}(\vec{u})=\exp \left\{-\frac{a^{2}}{2} \sum_{j=1}^{m} \beta_{j} u_{j}^{2}\right\}$ and let

$$
V_{m}(x)=f_{m}(\langle\vec{\alpha}, x\rangle) .
$$

Then $f_{m} \in \mathcal{S}\left(\mathbb{R}^{m}\right)$ and, by equation (4.7) and (4.8), we have

$$
\lim _{m \rightarrow \infty} V_{m}(x)=\exp \left\{-\frac{a^{2}}{2} \int_{0}^{T} x^{2}(t) d t\right\}
$$

for a.e. $x \in C_{0}[0, T]$.

The functional given by equation (4.8) is under our consideration and so we will apply the results obtain in previous sections to the functional $V_{m}$ given by equation (4.8).

Let $\Delta^{k} V_{m}(x)=\Delta^{k} f_{m}(\langle\vec{\alpha}, x\rangle)$. Then, using Theorem 4.1, the analytic Feynman integral of $\left[\Delta^{k} V_{m}\right]^{\wedge}$ exists and using equation (4.2), we have

$$
\begin{aligned}
& \int_{C_{0}[0, T]}^{\operatorname{anf}_{q}}\left[\Delta^{k} V_{m}\right]^{\wedge}(x) d m(x) \\
& =\left(\frac{\sqrt{-i q}}{2 \pi}\right)^{m} \sum_{l_{1}+\cdots+l_{m}=k} \frac{(-1)^{k} k !}{l_{1} ! \cdots l_{m} !} \prod_{j=1}^{m}\left(\frac{2 i}{q}\right)^{l_{j}} \frac{2 \Gamma\left(\frac{1}{2}+l_{j}\right)}{\sqrt{-2 i q}} \\
& \cdot \int_{\mathbb{R}} \exp \left\{-\frac{a^{2} \beta_{j}}{2} u_{j}^{2}-\frac{i u_{j}^{2}}{2 q}\right\}_{1} \mathcal{F}_{1}\left[-l_{j}, \frac{1}{2}, \frac{i u_{j}^{2}}{2 q}\right] d u_{j} .
\end{aligned}
$$

For examples, we compute the analytic Feynman integrals of the Fourier-type functional $\left[\Delta^{k} V_{m}\right]^{\wedge}$ as follows: 


\begin{tabular}{l|l}
\hline & $\int_{C_{0}[0, T]}^{\operatorname{anf}_{q}}\left[\Delta^{k} V_{m}\right]^{\wedge}(x) d m(x)$ \\
\hline$k=1(m=1)$ & $\frac{i}{4 q^{2} \sqrt{2}}\left(\frac{a^{2} \beta_{1}}{2}+\frac{i}{2 q}\right)^{-\frac{5}{2}}\left(\frac{i q a^{2} \beta_{1}}{2}-a^{4} \beta_{1}^{2} q-2 i a^{2} \beta_{1}\right)$ \\
\hline$k=2(m=1)$ & $\frac{1}{16 q^{4} \sqrt{2}}\left(\frac{a^{2} \beta_{1}}{2}+\frac{i}{2 q}\right)^{-\frac{5}{2}}$ \\
& $\cdot\left(12+\frac{12 i\left(a^{2} \beta_{1} q+i\right)^{2}}{q}-12\left(a^{2} \beta_{1} q+i\right)^{2}\right)$ \\
\hline$k=3(m=1)$ & $\frac{-15 i}{8 q^{5} \sqrt{2}}\left(\frac{\alpha^{2} \beta_{1}}{2}+\frac{i}{2 q}\right)^{-\frac{7}{2}}$ \\
& $\cdot\left(\alpha^{4} \beta_{1} q^{2}-\alpha^{4} \beta_{1} i q+\alpha^{2} \beta_{1} i q-\frac{2 i}{q}\right)$ \\
\hline
\end{tabular}

Table 4. Potential function in a Schrödinger equation

\section{REFERENCES}

1. R.A. Ewan: Then Cameron-Storvick operator-valued function space integrals for a class of finite-dimensional functionals. Thesis, University of Nebraska, 1973.

2. H.H. Bang: Functions with bounded spectrum. Trans. Amer. Math. Soc. 347 (1995), 1067-1080.

3. R.H. Cameron \& W.T. Martin: Fourier-Wiener transforms of analytic functionals belonging to $L_{2}$ over the space $C$ Duke Math. J. 14 (1947), 99-107.

4. __ : The orthogonal development of non-linear functionals in series of FourierHermite functionals. Ann. of Math. 48 (1947), 385-392.

5. R.H. Cameron \& D.A. Storvick: An $L_{2}$ analytic Fourier-Feynman transform Michigan Math. J. 23 (1976), 1-30.

6. __ Feynman integral of variation of functionals. in: Gaussian Random Fields, World Scientific, Singapore, 1980, 144-157.

7. __ Some Banach algebras of analytic Feynman integrable functionals. in: Analytic Functions, Kozubnik, 1979, Lecture Notes in Math. 798 Springer-Verlag, Berlin, 1980, $18-67$.

8. $\quad$ : Relationships between the Wiener integral and the analytic Feynman integral. Rend. Circ. Mat. Palermo (2) Suppl. 17 (1987), 117-133.

9. R.P. Feynman: Space-time approach to non-relativistic quantum mechanics. Rev. Modern Phys. 20 (1948), 115-142.

10. S.J. Chang, J.G. Choi \& D. Skoug: Evaluation Formulas for Conditional Function Space Integrals I. Stochastic Analysis and Applications 25 (2007), 141-168.

11. S.J. Chang \& H.S. Chung: Generalized Fourier-Wiener function space transforms. $J$. Korean Math. Soc. 46 (2009), 327-345. 
12. S.J. Chang, H.S. Chung \& D. Skoug: Convolution products, integral transforms and inverse integral transforms of functionals in $L_{2}\left(C_{0}[0, T]\right)$. Integral Transforms Spec. Funct. 21 (2010), 143-151.

13. Integral transforms of functionals in $L^{2}\left(C_{a, b}[0, T]\right)$. J. Four. Anal. Appl. 15 (2009), 441-462.

14. D.M. Chung \& S.J. Kang: Evaluation formulas for conditional abstract Wiener integrals. Stochastic Analysis and Applications 7 (1989), 125-144.

15. __ Evaluation formulas for conditional abstract Wiener integrals II. J. Korean Math. Soc. 27 (1990), 137-144.

16. H.S. Chung \& V.K. Tuan: Fourier-type functionals on Wiener space. to appear in the Bull. Korean Math. Soc.

17. __ Generalized integral transforms and convolution products on function space. Integral Transforms Spec. Funct. 22 (2011), 573-586.

18. _ _ A sequential analytic Feynman integral of functionals in $L_{2}\left(C_{0}[0, T]\right)$. to appear in the Integral Transforms Spec. Funct.

19. G.W. Johnson \& D.L. Skoug: Scale-invariant measurability in Wiener space. Pacific J. Math. 83 (1979), 157-176.

20. H.-H. Kuo: Gaussian Measure in Banach Space. Lecture Notes in Mathematics 463 Springer, Berlin.

21. V.K. Tuan: On the Paley-Wiener theorem. Theory of Functions and Applications. Collection of Works Dedicated to the Memory of Mkhitar M. Djrbashian. Yerevan, Louys Publishing House (1995), 193-196.

22. __ Paley-Wiener type theorems. Frac. Calc. Appl. Anal. 2 (1999), 135-143.

${ }^{a}$ Department of Mathematics, Dankook University, Cheonan 330-714, Korea

Email address: iylee@dankook.ac.kr

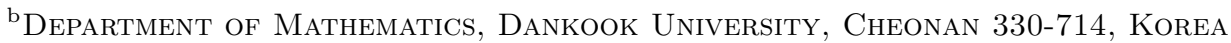

Email address: hschung@dankook.ac.kr

${ }^{\mathrm{c}}$ Department of Mathematics, Dankook University, Cheonan 330-714, Korea

Email address: sejchang@dankook.ac.kr 